\title{
A comparative study of the single-site laparoscopic herniorrhaphy using needle instruments and double-site laparoscopic herniorrhaphy in the minimally invasive treatment of inguinal hernias in children
}

\author{
FAN WANG, SUJUN LIU, YINGMO SHEN and JIE CHEN
}

\begin{abstract}
Hernia and Abdominal Wall Surgery, Beijing Chao-Yang Hospital of Capital Medical University, Beijing 100043, P.R. China
\end{abstract}
Received July 17, 2017; Accepted October 24, 2017

DOI: $10.3892 /$ etm.2018.5756

\begin{abstract}
The clinical effect of the single-site laparoscopic herniorrhaphy (SSLH) using needle instruments and doublesite laparoscopic herniorrhaphy (DSLH) in the treatment of inguinal hernias in children were compared. A total of 1,010 children with inguinal hernias who were treated in Chao-Yang Hospital from March 2011 to December 2014 were selected. According to the operation method, these children were divided into the single-site group using needle instruments $(n=508)$ and the traditional double-site group $(n=502)$. The clinical indexes of the two groups were compared. All the clinical indexes (operation time, intraoperative blood loss, time of off-bed and discharge time) in the single-site group using needle instruments were significantly lower than those in the traditional double-site group $(\mathrm{P}<0.001,<0.001$, $<0.001$ and $<0.003$, respectively), but the incidence rates of postoperative complications and contralateral occult hernia were at the similar level $(\mathrm{P}=0.249$ and 0.221 , respectively), so the difference was statistically insignificant. Patients in the two groups were followed up for 2 years and there was no recurrece in children in the single-site group while there was 2 in the double-site group. The SSLH using needle instruments is more effective with fewer traumas in the treatment of inguinal hernias in children than the DSLH. It is easier for children to recover from the herniorrhaphy with no scars, so it conforms more closely to the concept of minimally invasive herniorrhaphy, which can be promoted and applied for the treatment of inguinal hernias in children.
\end{abstract}

Correspondence to: Dr Jie Chen, Hernia and Abdominal Wall Surgery, Beijing Chao-Yang Hospital of Capital Medical University, 5 Jingyuan Road, Shi Jingshan, Beijing 100043, P.R. China

E-mail: chenjie6676@163.com

Key words: needle instruments, single-site laparoscopy, inguinal hernias in children, minimally invasive treatment

\section{Introduction}

Inguinal hernias in children is mainly caused by non-closed or incompletely closed congenital peritoneal encapsulation (1). It is a common disease frequently seen in children, which is considered a congenital disease. The high ligation of the hernias is the standard procedure for inguinal hernias in children with a high success and low incidence rate of complications (2). In previous years, with the development of the minimally invasive herniorrhaphy, the treatment method has been gradually changed from the traditional open into the minimally invasive laparoscopic herniorrhaphy. The meta-analysis of Yang et al (3) reported that the laparoscopic herniorrhaphy was more effective in the treatment of bilateral inguinal hernias with a lower incidence rate of contralateral hernias than conventional open herniorrhaphy. At the approach level, the laparoscopic herniorrhaphy has two approaches, that is; the intraperitoneal approach $(4,5)$; and the extraperitoneal approach (6). The extracorporeal approach refers to the operation that the suture is completed, the knot is tied and the inner ring is closed (by both single- and double-site laparoscopies) in vitro, which is the most widely used technology at present. In order to compare the difference between the two laparoscopic herniorrhaphies in the treatment of inguinal hernias in children, we selected 1,010 patients with inguinal hernias who were admitted to Beijing Chao-Yang Hospital of Capital Medical University from March 2011 to December 2014 and treated with the single-site laparoscopic herniorrhaphy (SSLH) and double-site laparoscopic herniorrhaphy (DSLH) for children with inguinal hernias.

\section{Patients and methods}

A total of 1,010 children with inguinal hernias who were diagnosed and treated by the western district of Beijing Chao-Yang Hospital of Capital Medical University were selected. A total of 502 children were treated with the traditional DSLH from March 2011 to June 2013 and 508 children were treated with the SSLH using needle instruments from July 2013 to the end of 2014. Symptoms and the results of ultrasound and other auxiliary tests of all the children were consistent with the diagnostic criteria of inguinal hernias (7). In the single-site 


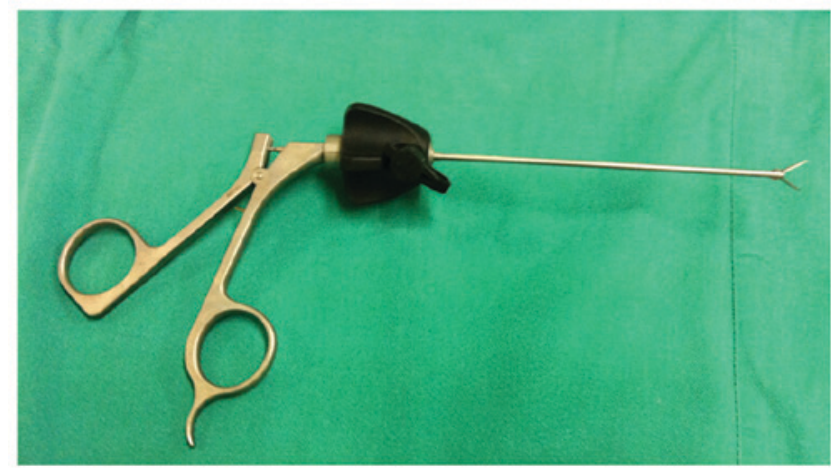

Figure 1. Miniature needle instrument.

group using needle instruments, there were 440 males and 68 females aged 1-11 years with the mean age of $4.32 \pm 1.62$ years. Among them, there were 371 children with hernias on the right side and 137 children on the left side. In the traditional doublesite group: There were 452 males and 50 females aged 1-11 aged years with the mean age of $4.46 \pm 1.34$ years. Among them, there were 356 children with hernias on the right side and 146 children on the left side. All the children were diagnosed with unilateral inguinal hernias and received the herniorrhaphy for the first time. Parents signed the informed consent. All the children were followed up for 2 years. The study was approved by the Ethics Committee of Beijing Chao-Yang Hospital of Capital Medical University and informed consents were signed by the patients and/or guardians.

\section{Operation methods}

The SSLH using needle instruments. Children received general anesthesia through the laryngeal mask airway and were placed in the trendelenburg position where the feet were higher than the head. A $5 \mathrm{~mm}$ incision was cut in the umbilical ring or the lower edge of the skin folds to establish pneumoperitoneum, and the air pressure was maintained at $8-10 \mathrm{mmHg}$ $(1 \mathrm{mmHg}=0.133 \mathrm{kPa})$. A $5 \mathrm{~mm}$ laparoscope was inserted at $30^{\circ}$ through puncturing the detection of bilateral inner ring. A
$1.5 \mathrm{~mm}$ incision was cut on the middle or relative upper point of the linea alba on the hypogastrium. A $1.5 \mathrm{~mm}$ incision was cut on the projection spot on the top surface of the inner ring, and the home-made needle instrument was inserted (Fig. 1). We punctured with no. 4 polyester thread crochet to the peritoneum. Under the laparoscopic monitoring, the needle instrument and crochet proceeded from 11 o'clock position of the inner ring to avoid the lower abdominal artery and along the inner ring from top to bottom under the peritoneum. After they came across seminiferous ducts and spermatic vessels, they punctured the peritoneum into the abdominal cavity. The needle instrument was used to pull the two threads, which were then left in the abdominal cavity, and the crochet was taken out. Afterwards, we inserted the crochet from the original skin incision. The crochet proceeded from the 12 o'clock position of the inner ring along the inner ring under the outer half cycle of peritoneum. After it reached the inner half cycle, we threaded the crochet with the abdominal suture and then took it out from the body. Then we squeezed the scrotum, and after the pneumatosis occurred at the groin area, we tightened the suture and knotted it in the skin. After that, we completed the high ligation of the hernias sac. Meanwhile, we detected the contralateral situation. If the contralateral hernias existed, we would conduct a herniorrhaphy at the same time. After the contralateral area was detected to be without error, we pulled out the puncture needle and removed the pneumoperitoneum to end the herniorrhaphy. Umbilical puncture holes were routinely sutured with a stitch, and the bio-adhesive bound the skin incision (Fig. 2).

The DSLH. Routine disinfection and draping were conducted. A $0.5 \mathrm{~cm}$ incision on the umbilical region was taken. After the establishment of pneumoperitoneum, the incision was inserted into the $5 \mathrm{~mm}$ trocar and then into the laparoscope lens. Then another $0.5 \mathrm{~cm}$ incision on the contralateral rectus abdominis was made, where a $5 \mathrm{~mm}$ trocar was inserted. A pair of operation forceps was inserted to probe the abdominal cavity and bilateral inner ring. Other operations were the same as those in the SSLH, and the herniorrhaphy was concluded.
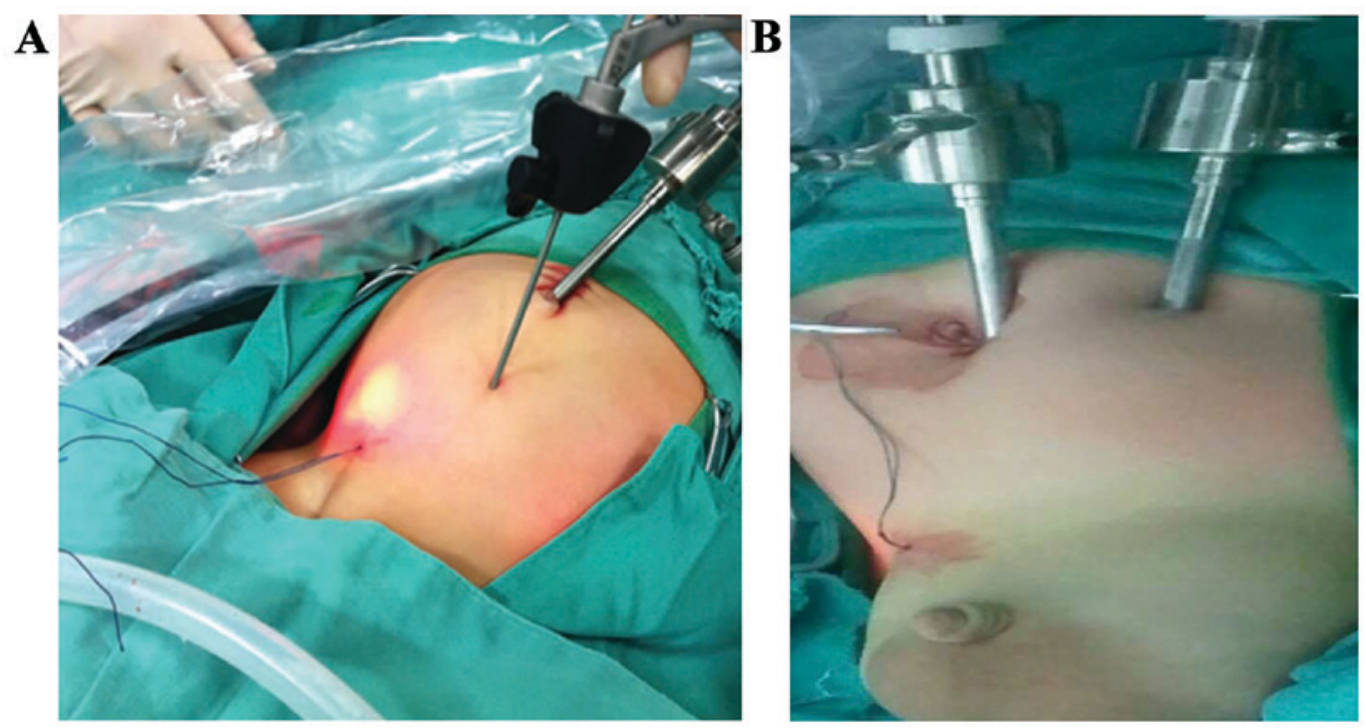

Figure 2. Comparison of the SSLH using needle instruments with the DSLH. (A) Single-site. (B) Double-site. SSLH, single-site laparoscopic herniorrhaphy. 
Table I. Comparisons of the clinical data of children with inguinal hernias in the SSLH group using needle instruments and the traditional double-site group.

\begin{tabular}{lccr}
\hline Parameters & $\begin{array}{c}\text { SSLH group using needle } \\
\text { instruments (n=508) }\end{array}$ & $\begin{array}{c}\text { Traditional DSLH group } \\
(\mathrm{n}=502)\end{array}$ & P-value \\
\hline Age & $4.32 \pm 1.62$ & $4.46 \pm 1.34$ & 0.446 \\
Sex & $440(86.6 \%)$ & $452(90 \%)$ & 0.090 \\
Male & $68(13.4 \%)$ & $50(10 \%)$ & 0.454 \\
Female & & & \\
Position & $371(73.0 \%)$ & $356(70.9 \%)$ & $<0.001$ \\
Right side & $137(27.0 \%)$ & $146(29.1 \%)$ & $<0.001$ \\
Left side & $6.33 \pm 1.56$ & $1.12 \pm 0.33$ & $<0.001$ \\
Operation time (min) & $0.58 \pm 0.15$ & $55(11.0 \%)$ & 0.003 \\
Intraoperative blood loss (ml) & & $447(89.0 \%)$ & 0.249 \\
Time of off-bed (h) & $483(95.1 \%)$ & $1.58 \pm 0.49$ & $5(1.0 \%)$ \\
$\quad<4$ & $25(4.9 \%)$ & $1(0.20 \%)$ & \\
$4-24$ & $1.23 \pm 0.16$ & $2(0.40 \%)$ & 0.221 \\
Discharge time (days) & $2(0.38 \%)$ & & \\
Complications & $1(0.19 \%)$ & $96(19.1 \%)$ & \\
Puncture infection & $1(0.19 \%)$ & & \\
Incision dehiscence & $02(0.40 \%)$ & & \\
Recurrence & $113(22.2 \%)$ & & \\
Appearance of contralateral occult hernia & & & \\
\hline SSLH, single-site laparoscopic herniornaphy. & & & \\
\hline
\end{tabular}

Statistical analysis. SPSS 17.0 (SPSS, Inc., Chicago, IL, USA) was used to analyze the data. The measurement data were expressed as mean $\pm \mathrm{SD}$. The independent two-sample t-test was conducted. The enumeration data were detected by Chi-square test. $\mathrm{P}<0.05$ was considered to indicate a statistically significant diference.

\section{Results}

As shown in Table I, among 1,010 children with inguinal hernias, there were 892 males and 118 females, in which there were 727 children with inguinal hernias at the right side and 283 children with inguinal hernias at the left side. A total of 508 children were treated with SSLH using needle instruments while 502 children underwent DSLH. There were no significant statistical differences $(\mathrm{P}=0.446,0.09$ and 0.454 , respectively) between the two groups in terms of sex, age, surgical site and other clinical data after comparisons, but these data were comparable. The operation time, intraoperative blood loss, time of off-bed and discharge time of the singlesite group were significantly lower than those of the traditional double-site group, so the differences were statistically significant $(\mathrm{P}<0.001,<0.001$ and $<0.01$, respectively). In terms of postoperative complications, as children in both groups were operated by skilled surgeons, there were no serious complications $(\mathrm{P}=0.249)$. There was 1 child with incision dehiscence in the single-site group and 2 children in the traditional double-site group. All children were followed up for 2 years. There was no recurrence in the single-site group, but 2 children had recurrence in the traditional double-site group. A total of 113 children were detected with contralateral occult hernia in the single-site group and 96 children in the traditional double-site group. The difference between the two groups has no statistical significance after the comparison $(\mathrm{P}=0.221)$. Intraoperative operations under operations of the laparoscope are shown in Fig. 3 and the postoperative comparison of effects is shown in Fig. 4. Abdominal scars were obvious in the double-site group.

\section{Discussion}

Laparoscopic herniorrhaphy in the treatment of children with inguinal hernias has been gradually replacing the traditional open high ligation of the hernias sac, which occupies an indispensable position. At present, there is no uniform norm for the inguinal hernias laparoscopic herniorrhaphy in China or other countries, but as the open herniorrhaphy, has been developed on the basis of the high ligation of the hernias sac combined with the advantages of the laparoscopic technology. Present laparoscopic herniorrhaphy methods and improved technologies exist in a wide range, so how to safely and effectively complete a herniorrhaphy with increasingly minimal traumas and no scars has become the new focal point of study. Based on this concept, we made a miniature needle instrument, which helps us operate the SSLH using the needle instrument. In the present study, we compared the new operation method SSLH using the needle instrument with the traditional DSLH in the prognosis of children with inguinal hernias so as to pinpoint the curative effect and safety of the SSLH using needle instruments for children with inguinal hernias. 

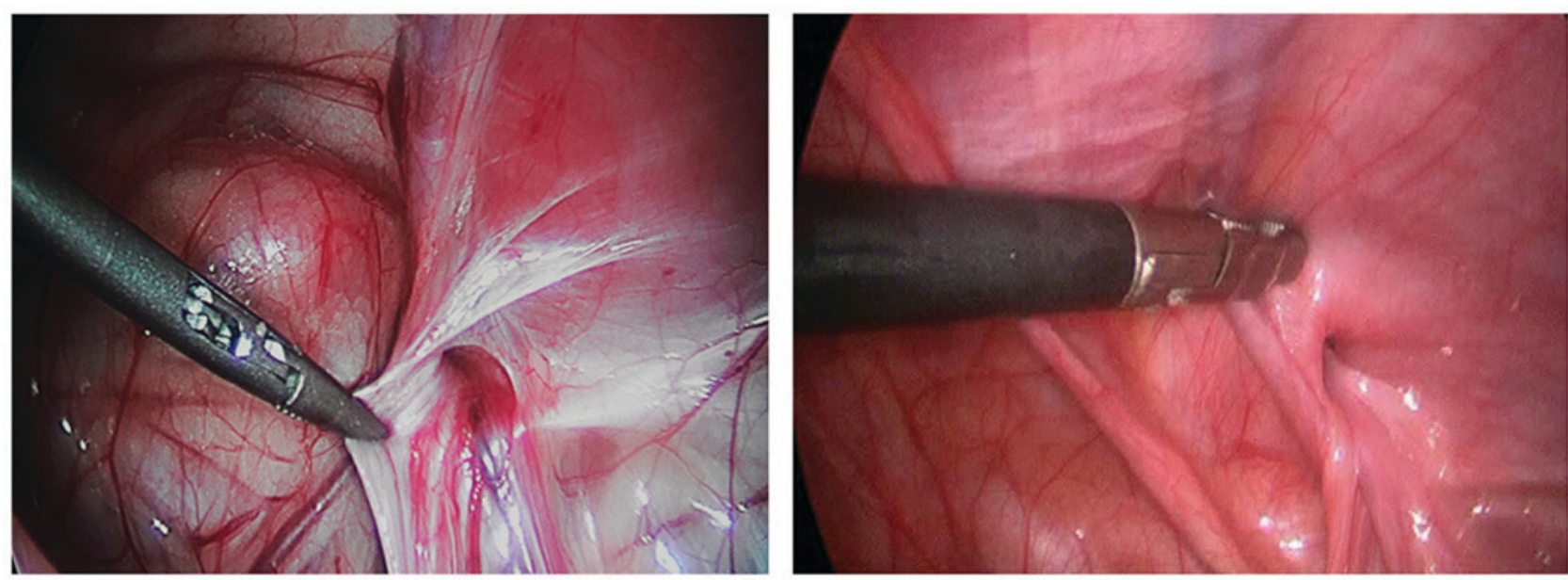

Figure 3. Comparison of the laparoscopic vision when pulling the peritoneum and positioning body surface between the SSLH using needle instruments with the DSLH. DSLH, double-site laparoscopic herniorrhaphy (left, single-site; right, double-site).
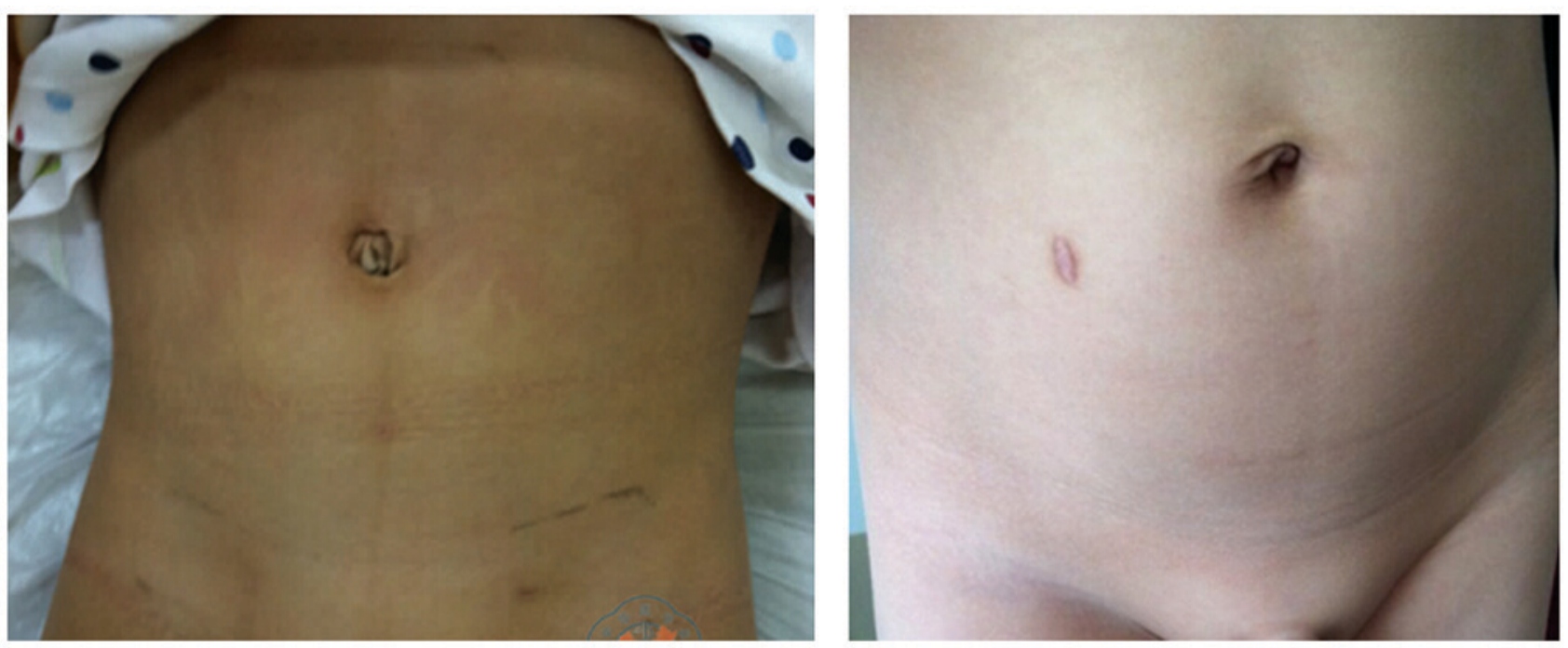

Figure 4. Comparison of the postoperative 1 month curative effect between the SSLH using needle instruments with the DSLH (left: single-site; right: double-site). SSLH, single-site laparoscopic herniorrhaphy; DSLH, double-site laparoscopic herniorrhaphy.

Results of our study showed that the operation time, intraoperative blood loss, time of off-bed and discharge time of the single-site group using needle instruments were significantly lower than those of the traditional double-site group (8), and the differences were statistically significant. As the needle instrument helped us quickly find the hernia sac as well as the peritoneum and the suture clamped by the needle holder, and reduced the time of placing a trocar and stitching a puncture, the operation time in the single-site group $(6.33 \mathrm{~min}$ on average) was shorter than that in the double-site group. In addition, children in the single-site group using needle instruments could move after waking up from anesthesia, and were discharged on the operation day without taking painkillers or antibiotics, greatly reducing the hospital stays in children. The umbilicus and linea alba approach of children: The needle instrument was punctured in the linea alba of the umbilicus, there appeared no muscle, nerve and vascular injury, and the bleeding was significantly reduced. Besides, children felt no pain caused by muscular traction and contraction, so it was easy for children to recover, and the time of off-bed of children was significantly shortened. As for complications, the SSLH using needle instruments had generalities of the laparoscopic herniorrhaphy, so the spermatic cord of the inner ring was not easy to be damaged under the direct vision. This herniorrhaphy retained the integrity of the inguinal canal, reduced the degree of nerve damage and effectively avoided the hematoma of scrotum and the recurrent hernias caused by neurocranial muscle paralysis. Due to children with low immunity congenitally, puncture infection would occur in some children to a certain degree and the incision dehiscence would occur in a very small number of children if the disinfection of the intraoperative umbilical incision was not strictly conducted and no antibiotics were used after herniorrhaphy. Another advantage of laparoscopic herniorrhaphy is that the contralateral occult hernia can be found and treated at once during the herniorrhaphy. About $20 \%$ children in the two groups were found to have contralateral occult hernia, and this herniorrhaphy avoided the pain caused by the re-herniorrhaphy. From the aesthetic aspects of herniorrhaphy, there was only a $5 \mathrm{~mm}$ observation hole left in the umbilical region in the single-site group using 
needle instruments, and there was basically no scar but the skin color was deep on the linea alba pinhole, which achieves the cosmetic results with no scar based on SSLH. Therefore, this herniorrhaphy is better than the traditional DSLH.

The technological difficulty of SSLH is that there is only the observation hole without the operation hole, but in order to overcome this technical bottleneck, a number of scholars attempted to improve new surgeries. Kimura et al (9) used port-closure techniques following laparoscopic herniorrhaphies; Zhou et al (10) selected umbilical approach herniorrhaphy, in which the umbilical cord incision was enlarged, and the laparoscope and the operating forceps were inserted at the same time; Shen et al (11) jointly applied ureteroscopic mirror body and the operation hole to conduct the herniorrhaphy, and moved the operation hole to the umbilical region close to the observation hole so as to avoid the appearance of scars. All these herniorrhaphies are not SSLH in the true sense. Moreover, the inserting site of laparoscope is close to that of the instrument, which results in the affection, interference and collision between each other as well as the existence of chopsticks effect. Difficulties also exist in this difficult operation, high technical threshold and the need for special equipment and expanded incision, which may also cause the corresponding complications, so it is not easy to be promoted. The single-site laparoscope can only puncture a $5 \mathrm{~mm}$ trocar hole on the umbilical ring and only a pinhole in the linea alba on the hypogastrium, so inserting a needle holder can help the operation forceps to pull the peritoneum and suture, making the herniorrhaphy operation simple and the learning curve short. This is more consistent with the concept of minimally invasive herniorrhaphy and easier to be popularized.

This study applied the improved SSLH using needle instruments for children with inguinal hernias for the first time. The curative efficacy of SSLH using needle instruments was better than that of the traditional DSLH, and it produced no scars. The SSLH is characterized by shorter operation time, fewer traumas, less pain, faster recovery period, more consistent with the concept of minimally invasive herniorrhaphy and easier to learn. Therefore, we recommend that this new herniorrhaphy should be clinically popularized and applied. However, longerterm curative efficacy also requires support from multi-center and large-sample data and longer-term follow-up.

\section{Competing interests}

The authors declare that they have no competing interests.

\section{References}

1. Potts WJ, Riker WL and Lewis JE: The treatment of inguinal hernia in infants and children. Ann Surg 132: 566-576, 1950

2. Dallas T: Treatment of congenital infuinal hernia. Ann Surg 135: 879-884, 1952.

3. Yang $\mathrm{C}$, Zhang $\mathrm{H}, \mathrm{Pu} \mathrm{J}$, Mei $\mathrm{H}$, Zheng $\mathrm{L}$ and Tong Q: Laparoscopic vs open herniorrhaphy in the management of pediatric inguinal hernias: A systemic review and meta-analysis. J Pediatr Surg 46:1824-1834, 2011.

4. Montupet $P$ and Esposito C: Laparoscopic treatment of congenital inguinal hernias in children. J Pediatr Surg 34: 420-423, 1999.

5. Zallen G and Glick PL: Laparoscopic inversion and ligation inguinal hernia repair in girls. J Laparoendosc Adv Surg Tech A 17: 143-145, 2007

6. Tatekawa Y: Laparoscopic extracorporeal ligation of hernia defects using an epidural needle and preperitoneal hydrodissection. J Endourol 26: 474-477, 2012.

7. Gilbert AI: An anatomic and functional classification for the diagnosis and treatment of inguinal hernias. Am J Surg 157: 331-333, 1989.

8. Liu SJ, Wang F, Zhu YL, Yang S, Shen YM, Wang MG and Chen J: Analysis of minimally invasive SSLH using needel instruments in the treatment of children with inguinal hernias. Chin Dep Herniorrhaph Abdom Wall Hernias 8: 17-20, 2014.

9. Kimura T, Yamauchi K, Ihara Y, Sawai T, Kosumi T and Yonekura T: Single-site laparoscopic herniorrhaphy using needle instruments for inguinal hernias in children: A novel technique. Surg Today 42: 100-103, 2012.

10. Zhou X, Song D, Miao Q and Shan W: Transumbilical endoscopic herniorrhaphy for completely enclosing inguinal hernias in children. J Pediatr Surg 46: 2417-2420, 2011.

11. Shen W, Ji H, Lu G, Chen Z, Li L, Zhang H and Pan J: A modified single-port technique for the minimally invasive treatment of pediatric inguinal hernias with high ligation of the vaginal process: The initial experience. Eur J Pediatr 169: 1207-1212, 2010.

This work is licensed under a Creative Commons Attribution-NonCommercial-NoDerivatives 4.0 International (CC BY-NC-ND 4.0) License. 